\title{
Emerged Coupling of Motor Control and Morphological Development in Evolution of Multi-Cellular Animats
}

\author{
Lisa Schramm ${ }^{1}$ and Yaochu Jin $^{2}$ and Bernhard Sendhoff ${ }^{2}$ \\ 1 Technische Universität Darmstadt, Karolinenplatz 5, 64289 Darmstadt, Germany \\ 2 Honda Research Institute Europe, Carl-Legien-Str. 30, 63073 Offenbach, Germany
}

\begin{abstract}
A model for co-evolving behavior control and morphological development is presented in this paper. The development of the morphology starts with a single cell that is able to divide or die, which is controlled by a gene regulatory network. The cells are connected by springs and form the morphology of the grown individuals. The movements of animats are resulted from the shrinking and relaxation of the springs connecting the lateral cells on the body morphology. The gene regulatory network, together with the frequency and phase shifts of the spring movements are evolved to maximize the distance that the animats can swim in a given time interval. To facilitate the evolution of swimming animats, a term that awards an elongated morphology is also included in the fitness function. We show that animats with different body-plans emerge in the evolutionary runs and that the evolved movement control strategy is coupled with the body plan.
\end{abstract}

\section{Introduction}

Brain-body co-evolution has attracted much attention in the research field of artificial life [1] since the seminal work of Karl Sims [2]. The most attractive aspect of the work is that a developmental model using a directed graph has been adopted for both neural controller and body plan. However, no significant progresses to understand biological principles have been made since Sims' work due to the following two facts. First, the power of the models for brainbody co-evolution remains practically unchanged $[3,4,5]$. A biologically plausible model should be able to describe the biological development of both nervous system and body plan. Whereas models for either detailed modeling of neural development [6] or morphology [7] have been suggested, few models can achieve a balanced depth in modeling the development of both neural controller and body plan, and most of them are not able to perform biologically meaningful behaviors. Second, most work on brain-body co-evolution was meant mainly for improving the efficiency of generating a specific behavior, rather than understanding biological principles. An exception has been the work by Bongard and Paul [8], which studied the correlation between morphological symmetry and locomotive efficiency. 
Most recently, increasing effects have been made to relate the research in brain-body co-evolution to biological principles. In [9], it is found that bilaterally symmetric body plan and neural architecture are favored in selection in a brain-body co-evolution of an elongated organism. The advantage of being able to evolve a bilaterally symmetric body plan or neural controller has been reported independently $[10,11]$. By taking energy efficiency into account in a hydra-like animat, it has been shown that a ring-like neural structure emerged in the animat [12], which is analogous to the nerve ring in biological hydra.

However, in the afore-mentioned models, the developmental process of the neural system and the body-plan is not included. To address this problem, we adapted a biologically plausible cell growth model [13] for neural development in a hydra-like animat with a fixed body plan [14]. In contrast to [6], the developed neural model is able to perform food grasping behavior by adjusting its connectivity and weights.

In this paper, we use a modified cell growth model for morphological development in a brain-body co-evolution environment, though behavior control is modeled in an abstract manner and evolved using a direct coding. Nevertheless, we believe that this work has made a solid step forward compared to [7] in that the developed morphology is able to perform a swimming behavior.

\section{The Computational Model}

The growth of the animat morphology is under the control of a gene regulatory network (GRN) and cellular physical interactions. The morphological development starts with a single cell put in the center of a two-dimensional computational area $(80 \mathrm{x} 80)$. Once the morphological development is complete, a controller is embedded in the morphology and the resulting swimming behavior is evaluated using a physics simulation engine.

\subsection{Chromosome for Morphological Development}

The cell growth model is slightly modified from the one in $[13,14]$. The model uses a GRN to regulate the developmental process. The GRN is defined by a set of genes consisting of regulatory units (RUs) and structural units (SUs). SUs define cellular behaviors, such as cell division and cell death, or the production of transcription factors (TFs) for intra- and inter-cellular interactions. The activations of the SUs are defined by the associated RUs, refer to Fig. 1. Note that single or multiple RUs may regulate the expression of a single or multiple SUs and that RUs can be activating $\left(R U^{+}\right)$or repressive $\left(R U^{-}\right)$.

Each RU and TF has a certain affinity value that determines whether a TF can influence a RU. If the difference between the affinity values of a TF and a $\mathrm{RU}$ is smaller than a predefined threshold $\epsilon$ (in this work $\epsilon$ is set to 0.2 ), the TF can be bound to the RU to regulate. The affinity overlap $\left(\gamma_{i, j}\right)$ between the $i$-th $\mathrm{TF}$ and $j$-th RU is defined by:

$$
\gamma_{i, j}=\max \left(\epsilon-\left|\operatorname{aff}_{i}^{\mathrm{T} F}-\operatorname{aff}_{j}^{\mathrm{R} U}\right|, 0\right) .
$$




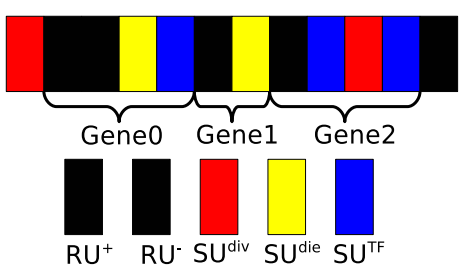

Fig. 1. An example chromosome for the development.

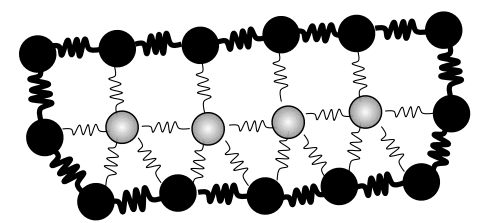

Fig. 2. Illustration of a body plan consisting of cells connected by springs. The springs at the outside of the body are able to change their natural length.

If $\gamma_{i, j}$ is greater than zero and the concentration $c_{i}$ of the $i$-th TF is above a threshold $\left(\vartheta_{j}\right)$ defined in the $j$-th RU, then the $i$-th TF influences the $j$-th RU.

Thus, the activation level contributed by this $\mathrm{RU}$ (denoted by $a_{j}, j=1, \ldots, N$ ) sums up to $a_{j}=\sum_{i=1}^{M}\left|c_{i},-\vartheta_{j}\right|$, where $M$ is the number of existing TFs. The expression level of the $k$-th gene, that is regulated by $N$ RUs, can be defined by

$$
\alpha_{k}=100 \sum_{j=1}^{N} h_{j} a_{j}\left(2 s_{j}-1\right),
$$

where $s_{j} \in(0,1) .2 s_{j}-1$ denotes the sign (positive for activating and negative for repressive) of the $j$-th $\mathrm{RU}$ and $h_{j}$ is a parameter representing the strength of the $j$-th RU. If $\alpha_{k}>0$, then the $k$-th gene is activated and its corresponding behaviors coded in the SUs are performed.

An SU that produces a TF ( $\mathrm{SU}^{\mathrm{TF}}$ ) encodes all parameters related to the TF, such as the affinity value, the decay rate $D_{i}^{c}$, the diffusion rate $D_{i}^{f}$, as well as the amount of the TF to be produced:

$$
A=\beta \frac{2}{1+e^{-20 \cdot f \cdot \alpha}}-1
$$

where $f$ and $\beta$ are both encoded in the $\mathrm{SU}^{\mathrm{TF}}$.

A TF produced by a SU can be partly internal and partly external. To determine how much of a produced TF is external, a percentage $\left(p^{\text {ex }} \in(0,1)\right)$ is also encoded in the corresponding gene. Thus, $p^{\mathrm{ex}} \cdot A$ is the amount of external $\mathrm{TF}$ and $\left(1-p^{\mathrm{ex}}\right) \cdot A$ is that of the internal TF.

External TFs are put on four grid points around the center of the cell, which undergo first a diffusion and then a decay process:

$$
\begin{aligned}
\text { Diffusion: } & \mathbf{u}_{i}(t)=\mathbf{u}_{i}(t-1)+0.1 \cdot D_{i}^{f} \cdot\left(\mathbf{G} \cdot \mathbf{u}_{i}(t-1)\right), \\
\text { Decay: } & \mathbf{u}_{i}(t)=\min \left(\left(1-0.1 \cdot D_{i}^{c}\right) \mathbf{u}_{i}(t), 1\right),
\end{aligned}
$$

where $\mathbf{u}_{i}$ is a vector of the concentrations of the $i$-th TF at all grid points and the matrix $\mathbf{G}$ defines which grid points are adjoining.

In our experiments we put two prediffused, external TFs without decay and diffusion in the computation area. The first TF has a constant gradient in the $\mathrm{x}$-direction and the second in the $\mathrm{y}$-direction. 


\begin{tabular}{|l|l|l|l|}
\hline Mass of cells $m$ & 0.5 & Short natural length of springs $l_{s}$ & 1.2 \\
\hline Radius of cells $r$ & 0.5 & Minimal periodic time $T_{\min }$ & 10 \\
\hline Damping constant $d$ & 1 & Maximal periodic time $T_{\max }$ & 400 \\
\hline Spring strength $c$ & 5 & Simulation length $t_{\text {sim }}$ & 300.0 \\
\hline Normal natural length of springs $l_{n}$ & 2 & & \\
\hline
\end{tabular}

Table 1. Constants for the mechanical simulation environment

The SUs encode cellular behaviors and the related parameters. The SU for cell division encodes the angle of division, indicating where the daughter cell is placed. A cell with an activated SU for cell death dies at the end of the developmental timestep.

\subsection{Chromosome for Motor Control}

To embed a motor controller into the developed morphology, cells must be connected to a whole body plan. Cells are connected with a damped spring if the distance between them is smaller than 2.5. If a cell has fewer than two connections, it is then connected to its nearest neighbor to ensure morphological stability, see Fig. 2. The mechanical setups of the cells and springs are listed in Table 1.

The movement is defined by a change in the natural length of the springs connecting the lateral cells, as depicted in bold in Fig. 2. To ease the movement, the cell radius is set to 0.5 so that there is sufficient space between the cells for them to move. The natural length of the springs switches between $l_{n}$ and $l_{s}$ within one period $T$, which is subject to evolution. The morphology is split into 24 predefined segments and all springs in the same segment have the same phase shift $(\rho \in[0, T])$.

\subsection{Physics Simulation Engine}

We use BREVE [15] to simulate the behavior of the animats. In addition, we use a simple model for simulating the effects of water forces, which has also been adopted in [16]. In this model, the water forces for different elements $i$ are computed as follows:

$$
\begin{aligned}
\boldsymbol{F}^{i} & =\boldsymbol{F}_{T}^{i}+\boldsymbol{F}_{N}^{i}, \\
\boldsymbol{F}_{T}^{i} & =-\lambda_{T} \cdot \operatorname{sgn}\left(\boldsymbol{v}_{T}^{i}\right) \cdot\left(\boldsymbol{v}_{T}^{i}\right)^{2}, \\
\boldsymbol{F}_{N}^{i} & =-\lambda_{N} \cdot \operatorname{sgn}\left(\boldsymbol{v}_{N}^{i}\right) \cdot\left(\boldsymbol{v}_{N}^{i}\right)^{2},
\end{aligned}
$$

where $\lambda_{T}$ and $\lambda_{N}$ are the drag coefficients for each direction. $\lambda$ depends on the effective area, a shape coefficient of the element and the fluid density. $\boldsymbol{v}_{T}^{i}$ and $\boldsymbol{v}_{N}^{i}$ are the velocities of element $i$ in normal and tangential direction. We set $\lambda_{T}=0.001$ and $\lambda_{N}=2.5$ in this work. The water forces are computed for cells 
in the lateral of the body plan, represented by black circles in Fig. 2. The normal and tangential vectors of the body parts ( $i$-th sphere) can be calculated by:

$$
\begin{aligned}
\boldsymbol{t}^{i} & =\frac{\boldsymbol{p}^{i-1}-\boldsymbol{p}^{i+1}}{\left|\boldsymbol{p}^{i-1}-\boldsymbol{p}^{i+1}\right|} \\
\boldsymbol{n}^{i} & =\left(\begin{array}{cc}
0 & -1 \\
1 & 0
\end{array}\right) \cdot \boldsymbol{t}^{i}
\end{aligned}
$$

where $\boldsymbol{p}^{i}$ is the position vector of the $i$-th cell and $\boldsymbol{p}^{i-1}$ and $\boldsymbol{p}^{i+1}$ are the positions of the neighboring cells at the outer side of the morphology.

$$
\begin{aligned}
& \boldsymbol{v}_{N}^{i}=\boldsymbol{n}^{i} \cdot \boldsymbol{v}^{i}, \\
& \boldsymbol{v}_{T}^{i}=\boldsymbol{t}^{i} \cdot \boldsymbol{v}^{i},
\end{aligned}
$$

where $\boldsymbol{v}^{i}$ is the velocity of the $i$-th cell.

\section{Evolution of Swimming Behaviors}

An extended $(\mu, \lambda)$ evolution strategy with individual strategy parameter adaptation has been employed in this work. The strategy parameters $(\sigma)$ are bounded to $\sigma_{m} \in\left[1 e^{-6}, 1 e^{-4}\right]$ and $\sigma_{c} \in\left[1 e^{-6}, \infty\right]$ for the chromosome for morphological development and that for behavior control, respectively. For the chromosome for morphological development, we also use gene duplication and transposition in addition to mutations. In one setup, gene deletion is also applied, refer to Table 2 .

We minimize the following fitness function:

$$
f=f_{\text {swim }}+f_{\text {shape }},
$$

where $f_{\text {swim }}$ defines the distances between the center of masses of the body plan at the beginning and the end of the simulation:

$$
f_{\text {swim }}=-\left|\left(\sum_{i=0}^{n} \boldsymbol{x}^{i}(t=0)\right)-\left(\sum_{i=0}^{n} \boldsymbol{x}^{i}\left(t_{\text {end }}\right)\right)\right|
$$

and $f_{\text {shape }}$ awards elongated shapes:

$$
\begin{aligned}
f_{\text {shape }}= & \max \left\{\min _{i}\left\{\boldsymbol{x}^{i}(0)\right\},-30\right\}-\min \left\{\max _{i}\left\{\boldsymbol{x}^{i}(0)\right\}, 30\right\} \ldots \\
& \ldots-\min \left\{\min _{i}\left\{\boldsymbol{x}^{i}(1)\right\},-5\right\}+\max \left\{\max _{i}\left\{\boldsymbol{x}^{i}(1)\right\}, 5\right\},
\end{aligned}
$$

where the best reachable value for $f_{\text {shape }}$ is -50 .

To limit the computational cost, a maximum of 501 cells is allowed. If this number is exceeded before 20 iterations, the developmental process will be stopped. To have a meaningful morphology, the number of cells should be larger than 10. In case that the number of cells is larger than 500 or smaller than 10 , a strong penalty is applied.

In the experiments, we set $\mu=30$ and $\lambda=200$. The developmental process is computed for $t_{d e v}=20$ iterations. We run the evolution with three slightly different setups, which are listed in Table 2. 


\begin{tabular}{|l|l|l|l|l|l|}
\hline & initial \# RUs & initial \# SUs & $p_{\text {transp }}$ & $p_{\text {dup }}$ & $p_{\text {del }}$ \\
\hline Setup 1 & 66 & 54 & 0.05 & 0.05 & 0 \\
\hline Setup 2 & 30 & 30 & 0.05 & 0.05 & 0 \\
\hline Setup 3 & 30 & 30 & 0.05 & 0.02 & 0.03 \\
\hline
\end{tabular}

Table 2. EA setups
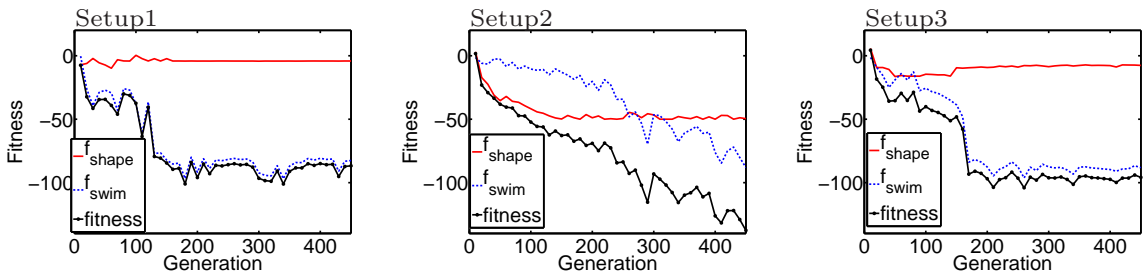

Fig. 3. Fitness profiles from the three setups, which are plotted every 10 generations.

\section{Results and Analysis}

In the following, we analyze the fitness profile, the morphology and the control strategy of three animats, picked out from generation 1518, 541 and 197 in the three setups, respectively. We have plotted the fitness for swimming behavior $f_{\text {swim }}$ and that for morphology $f_{\text {shape }}$ separately every 10 generations in Fig. 3. The $f_{\text {shape }}$ of the three animats are $-4.21,-50.0$, and -9.42 , respectively, while the $f_{\text {swim }}$ are $-97.38,-107.50$, and -84.87 , respectively. From Fig. 3 , we can see that the "fitnesses for shape" in both setups 1 and 3 have converged to a local minimum around generation 150. As a result, the morphology from these two setups is much smaller than that obtained from setup 2, refer to Fig. 4. Another observation is that the fitness for swimming in setup 2 improves steadily in 450 generations and is better than that from setups 1 and 3. Actually, the best animat evolved in setup 2 reached the border of the simulation area within the predefined simulation time.

More interestingly, we found that animats have also evolved different strategies for swimming. In setups 1 and 3, where the evolved morphology is very short, a control period of $T=10$ is evolved. By contrast, a period $T=16.3$ has been evolved for the animat in setup 2. In other words, the frequency of the rhythmic movement of the shorter animats is much faster than that of the large one, which makes sense for improving the swimming efficiency. In addition, different phase
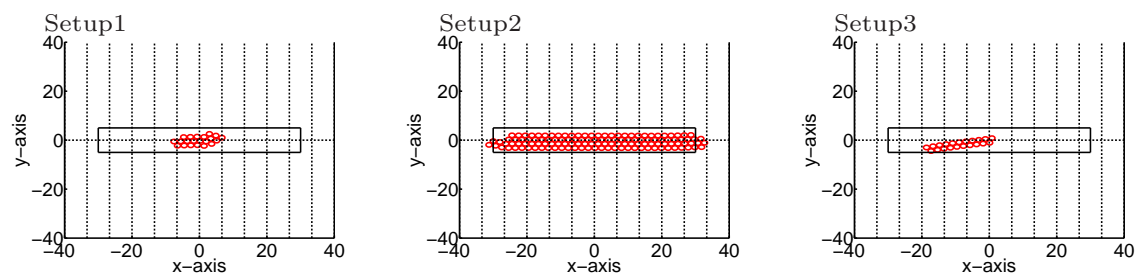

Fig. 4. Morphologies of the three individuals. 


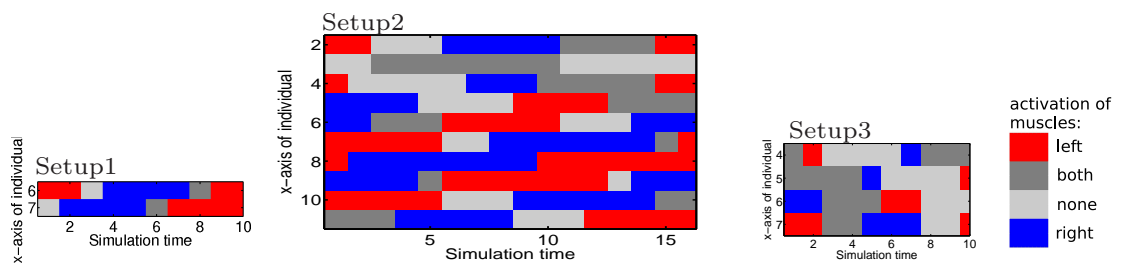

Fig. 5. Evolved phase coordination strategies of the three individuals.

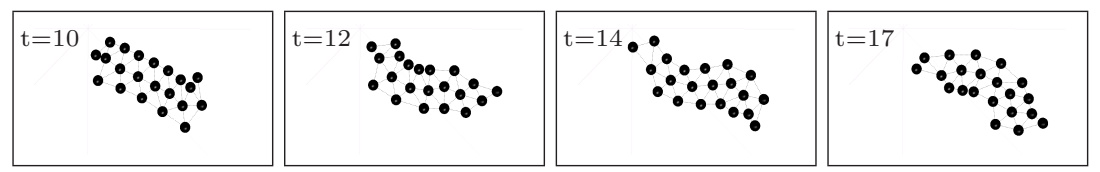

Fig. 6. Snapshots of the movement of the analysed individual from setup 1 .

coordination strategies have also been evolved for the three animats, as shown in Fig. 5. From the phase shift patterns, we can see that animats from setups 1 and 2 produce undulatory movements, while the animat from setup 3 generates peristaltic movements, similar to a caterpillar. A few snapshots of the resulting movement patterns of the three animats are presented in Fig. 6, Fig. 7, and Fig. 8, respectively (videos are available at www.rtr.tu-darmstadt.de/coevolution).

\section{Conclusion and Future Work}

We have presented a model for co-evolving morphological development and motor control for swimming animats. The morphological development is based on a cellular growth model regulated by a GRN, whilst the motor control is represented by the period and phase shifts of the springs. Compared to the direct graph model used by Sims [2] and the L-system by Horn and Pollak [3], our model for morphological development is biologically more plausible. From three slightly different setups of the evolutionary algorithm, three swimming patterns have emerged, which are adapted to the different morphologies. As far as we know, this is the first work to present a gene-regulated multi-cellular model for morphological development of animats that can perform a functional behavior and to disclose a coupling between the motor control strategy and the body plan.

In future work, we will include a GRN-based model for neural development so that both the neural system and body morphology are subject to a developmental process. By investigating brain-body co-evolution with such biologically plausible

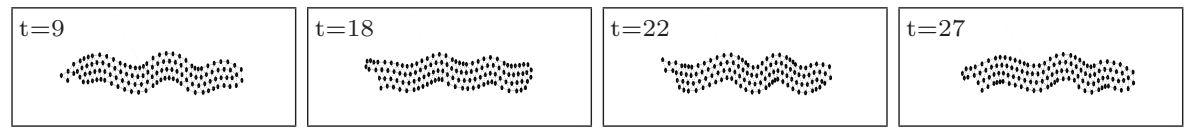

Fig. 7. Snapshots of the movement of the analysed individual from setup 2. 
VIII

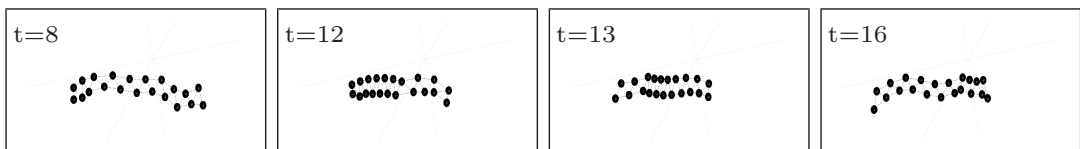

Fig. 8. Snapshots of the movement of the analysed individual from setup 3.

models, we hope to gain deeper insights into the co-evolution of nervous systems and morphologies in biology.

Acknowledgments We would like to thank T. Steiner, M. Olhofer, D. Weiler and N. Einecke for inspiring discussions.

\section{References}

1. Taylor, T., Massey, C.: Recent developments in the evolution of morphologies and controllers for physically simulated creatures. Artificial Life 7(1) (2001) 77-87

2. Sims, K.: Evolving virtual creatures. In: Proceedings SIGGRAPH. (1994) 15-22

3. Hornby, G., Pollack, J.: Evolving L-systems to generate virtual creatures. Computers \& Graphics 25 (2001) 1040-1048

4. Miconi, T., Channon, A.: An improved system for artificial creatures evolution. In: Proceedings of Artificial Life X. (2006) 255-261

5. Spector, L., Klein, J., Feinstein, M.: Division blocks and the open-ended evolution of development, form, and behavior. In: Proceedings of GECCO. (2007) 316-323

6. Kitano, H.: A simple model of neurogenesis and cell differentiation based on evolutionary large-scale chaos. Artificial Life 2(1) (1995) 79-99

7. Eggenberger, P.: Evolving morphologies of simulated 3d organisms based on differential gene expression. In: Proceedings of Artificial Life IV. (1997) 205-213

8. Bongard, J.C., Paul, C.: Investigating morphological symmetry and locomotive efficiency using virtual embodied evolution. In: Proc. of SAB. (2000) 420-429

9. Jones, B., Jin, Y., Sendhoff, B., Yao, X.: Evolving functional symmetry in a three dimensional model of an elongated organism. In: Artificial Life XI. (2008) 305-312

10. Mazzapioda, M., Cangelosi, A., Nolfi, D.: Co-evolving morphology and control: A distributed approach. In: Congress on Evolutionary Computation. (2009) In press.

11. Oros, N., Steuber, V., Davey, N., Canamero, L., Adams, R.: Evolution of bilateral symmetry in agents controlled by spiking neural networks. In: IEEE Symposium on Artificial Life, IEEE Press (2009) 116-123

12. Jones, B.H., Jin, Y., Yao, X., Sendhoff, B.: Evolution of neural organization in the hydramat. In: Proc. of ICONIP 2008, Springer (2008) 216-223

13. Steiner, T., Schramm, L., Jin, Y., Sendhoff, B.: Emergence of feedback in artificial gene regulatory networks. In: Proc. of CEC. (2007) 867-874

14. Jin, Y., Schramm, L., Sendhoff, B.: A gene regulatory model for the development of primitive nervous systems. In: Proc. of ICONIP 2008, Springer (2008) 48-55

15. Klein, J.: BREVE simulation environment. Open-Source Software Package. Source code available at: http://www.spiderland.org/.

16. Sfakiotakis, M., Tsakiris, D.: Simuun: A simulation environment for undulatory locomotion. International Journal of Modelling and Simulation 26(4) (2006) 350358 